\title{
Parallelization of thermochemical nanolithography $\dagger$
}

\author{
Keith M. Carroll, ${ }^{a}$ Xi Lu, ${ }^{a}$ Suenne Kim, ${ }^{\text {ab }}$ Yang Gao, ${ }^{a}$ Hoe-Joon Kim, ${ }^{c}$ Suhas Somnath, ${ }^{c}$ \\ Laura Polloni, ${ }^{\text {de }}$ Roman Sordan, ${ }^{d}$ William P. King, ${ }^{c}$ Jennifer E. Curtis ${ }^{\star a}$ \\ and Elisa Riedo*a
}

Received 25th October 2013

Accepted 24th November 2013

One of the most pressing technological challenges in the development of next generation nanoscale devices is the rapid, parallel, precise and robust fabrication of nanostructures. Here, we demonstrate the possibility to parallelize thermochemical nanolithography (TCNL) by employing five nano-tips for the fabrication of conjugated polymer nanostructures and graphene-based nanoribbons.

Nanofabrication is the process of making functional structures with arbitrary patterns having nanoscale dimensions. ${ }^{\mathbf{1} 4}$ Nanofabrication has been widely implemented commercially for improving microelectronic devices and information technology, to increase the density of components, to lower their cost, and to increase their performance per device and per integrated circuit. ${ }^{5}$ Other areas of applications beyond information processing and storage include optics, ${ }^{6}$ cell biology, ${ }^{7}$ and biomedicine. $^{8}$

Two widely used methods in industry nowadays are photolithography and particle beam lithography. The limitations of these conventional approaches, such as limitations in resolution, high capital and operational costs, limited flexibility in terms of materials which can be patterned and fabricated, have motivated the development of unconventional fabrication techniques such as soft lithography, self assembly, and scanning probe lithography ${ }^{3,7}$ (SPL). One of the main limitations in the different SPL methods is that the throughput is limited by the usually slow and serial writing process.

${ }^{a}$ School of Physics, Georgia Institute of Technology, 837 State Street, Atlanta, Georgia 30332-0430,USA.E-mail: jennifer.curtis@physics.gatech.edu; riedo@gatech.edu ${ }^{b}$ Department of Applied Physics, Hanyang University, Ansan 426-791, South Korea ${ }^{c}$ Department of Mechanical Science and Engineering, University of Illinois at UrbanaChampagne, 1206 West Green Street, Urbana, Illinois 61801-2906, USA

${ }^{d} L$-NESS, Department of Physics, Politecnico di Milano, Via Anzani 42, 22100 Como, Italy

${ }^{e}$ Department of Science and High Technology, Universitá degli Studi dell'Insubria, Via Valleggio 11, 22100 Como, Italy

$\dagger$ Electronic supplementary information (ESI) available: Details on the cantilevers array, on the sample preparation, and on the GO AFM experiments.
A practical approach to SPL for high-volume, parallel production may emerge by simultaneously writing nanostructures with multiple probes. Various designs of atomic force microscopy (AFM) probe arrays have been developed for applications in different AFM-based lithography techniques. While the parallelization in AFM-based lithography is at its infancy, some important advances have been made, such as the thermal probes array, also called Millipede, ${ }^{9}$ for thermomechanical writing of topographical structures in polymers, the dip-pen nanolithography (DPN) probes array ${ }^{10}$ for delivering inks through AFM probes, and the electro-oxidizing tip array to pattern silicon. ${ }^{\mathbf{1 1}}$

The most prominent challenges for parallelization of any SPM/AFM based lithography are the cost, the ability to image and simultaneously to write on a substrate, the resolution and reliability, as well as the possibility to use the same instrument for a variety of material applications.

Recently, it has been shown that thermochemical nanolithography ${ }^{\mathbf{1 2 , 1 3}}$ (TCNL) is a versatile AFM-based technique that can be used to fabricate nanostructures and nanopatterns of graphene-based materials, ${ }^{\mathbf{1 4 , 1 5}}$ piezoelectric/ferroelectric ceramics, ${ }^{16}$ polymers, ${ }^{17-21}$ proteins, ${ }^{22}$ and DNA. ${ }^{22}$ Furthermore, TCNL was demonstrated to be capable to write sub-micron gradients of amine groups on polymers. ${ }^{23}$ Other variants of thermally activated reactions include work on retro diels alder reactions $^{\mathbf{2 4 , 2 5}}$ for 3-D patterning, and work on co-polymers, ${ }^{26}$ where thermal cantilevers were employed to study and modify copolymers.

TCNL uses a thermal probe to locally heat the surface of a material. ${ }^{11}$ This heat produces a nano-scale chemical or physical transformation which can be controlled in terms of spatial resolution and extent of chemical conversion. Very importantly, TCNL speed of writing is only limited by the speed of the heat transfer and the chemical reaction. So far, writing speeds up to $\mathrm{mm} \mathrm{s}^{-1}$ for a single tip have been demonstrated. The aforementioned Millipede operates on the principles of thermomechanical lithography where the heat is used to locally melt or modify the topography of a material such as a polymer. 
Differently, TCNL induces chemical reactions in order to chemically pattern substrates. In this sense, DPN and TCNL have more similar goals. Both methods aim at fabricating nanostructures made of a material that is different from the rest of the substrate. TCNL activates a chemical reaction and it is based on heat transfer. DPN directly deposit new material and it is based on mass transfer. Advantages and disadvantages ultimately depend on the type of application and material to be nanofabricated.

Here, we demonstrate the parallelization of TCNL by using a five-tip array. The same array is used in situ to write and image microstructures, nanowires, and complex patterns of a conjugate luminescent semiconducting polymer, as well as conductive nanoribbons of reduced graphene oxide. Resolution down to sub-50 $\mathrm{nm}$ over areas of $500 \mu \mathrm{m}$ and parallel complex 3D-patterning are demonstrated.

\section{Results and discussion}

Fig. 1a depicts a cartoon of a thermal tip array containing five cantilevers with the corresponding nano-tips, spaced about 110 $\mu \mathrm{m}$ each other. For convenience, we label the tips 1 through 5 . Each cantilever in the array ${ }^{27}$ is joule-heated as previously reported in literature for single cantilevers, ${ }^{28}$ and it is individually addressable with a voltage bias, which is used to control the current flowing in the cantilever to achieve the joule-heating at the resistive tip. Recent experiments ${ }^{27}$ show that these probes arrays can image surfaces with $0.6 \mathrm{~nm}$ vertical resolution, and can be used for thermomechanical lithography to pattern topographic variations into a fluorocarbon film. More

(a)

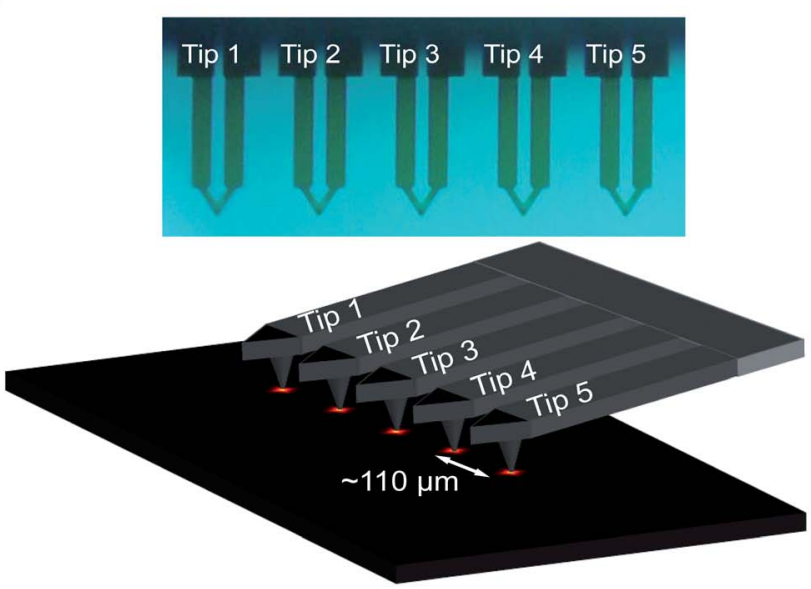

(c)

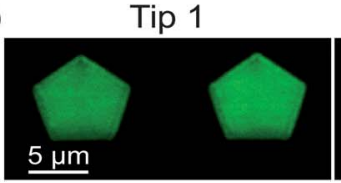

Tip 2
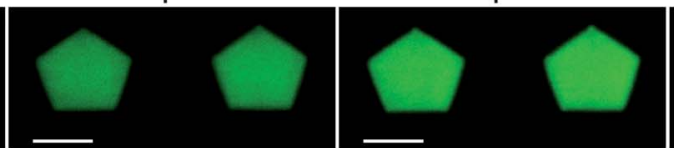

Tip 3

(b)

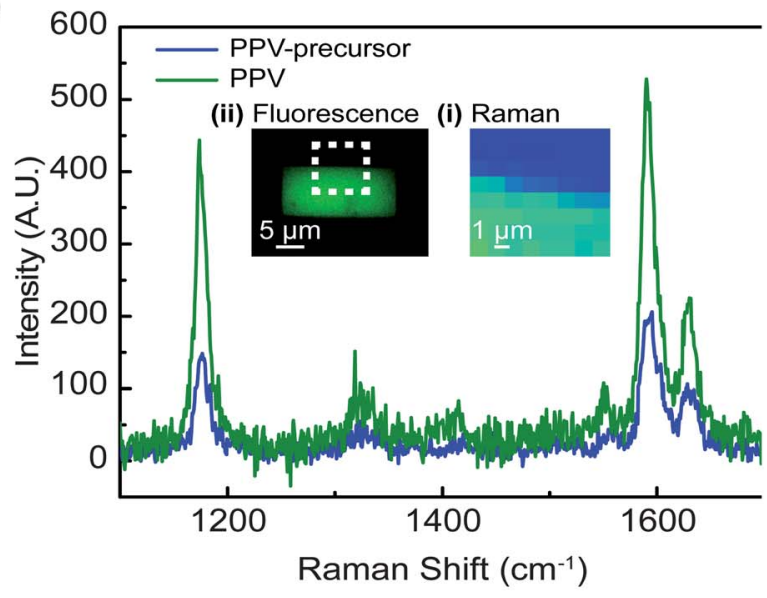

Tip 4

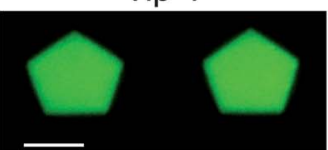

Tip 5

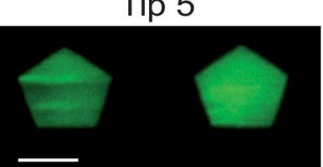

Fig. 1 (a) Optical Image and cartoon of the five thermal cantilevers and tips array for parallel TCNL. (b) Raman spectrum of PPV precursor (blue) and a TCNL produced pattern of PPV (green). In the inset we show the corresponding Raman image (I) and fluorescence image (II) of the precursor film and PPV pattern. (c) Fluorescence image of five PPV pentagonal double-patterns produced by the TCNL array and consisting each of two pentagons produced with two different temperatures. 
The parallel writing capability of the TCNL array is then demonstrated in Fig. 1c, where we show five almost identical fluorescent images of two PPV pentagons produced with the five tips array. Each tip was used to write two PPV pentagons with two different temperatures $\left(T_{1}\right.$ and $\left.T_{2}\right)$, at a linear speed per tip of $10 \mu \mathrm{m} \mathrm{s}^{-1}$. The images were taken with an epi-fluorescent microscope at an excitation of 488 and emission filter centered around $535 \mathrm{~nm}$; this excitation/emission filters are consistent with the photoluminescent spectrum of PPV. As the heater temperature increases moving from the first to the second pentagon in all the five tips patterns, it is possible to observe an increase in the fluorescence intensity signal consistent with an increased amount of precursor that becomes PPV. We note that the five double-patterns are not completely identical. This is due to the fact that the tips are not perfectly aligned and therefore they are contacting the precursor film at different loads. A way to overcome and control this problem is to calibrate and control the heat provided to each tip individually, in order to guarantee the same amount of heat transferred at the tip-sample contact.

Having demonstrated the ability to convert the precursor film to PPV, we performed a series of experiments (see Fig. 2) to demonstrate the ability of the array to perform TCNL with nanoscale resolution. We wrote a set of PPV lines with eight increasing temperatures from left to right for each tip. For this experiment, the surfaces were spun cast instead of drop cast. Fig. 2a shows fluorescent images, each representing eight PPV wires written by each tip with increasing temperature; again we observe that as the temperature is increased (left to right) more of the precursor undergoes the transformation in PPV. Fig. $2 b$ shows the corresponding AFM topography images for the wires produced by each tip. We show in Fig. 2c five fluorescence images of five Mona Lisa PPV structures produced by a TCNL
Tip 1

(a)

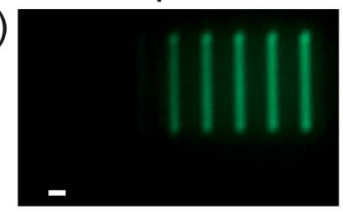

Tip 2

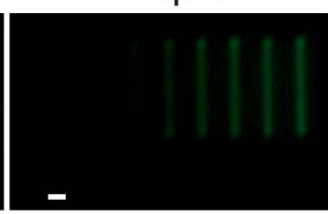

Tip 3

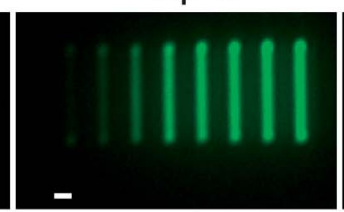

Tip 4

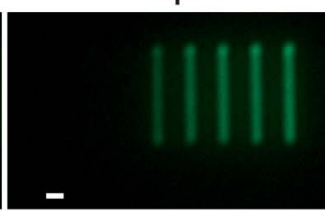

Tip 5

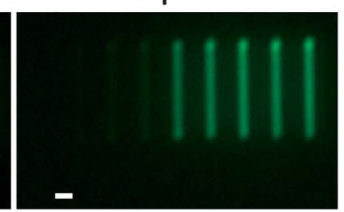

(b)
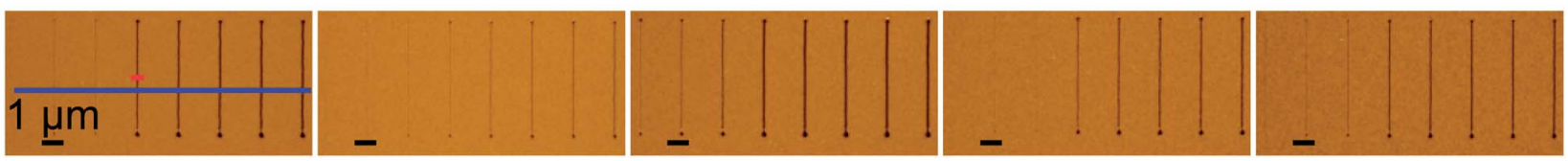

(c)
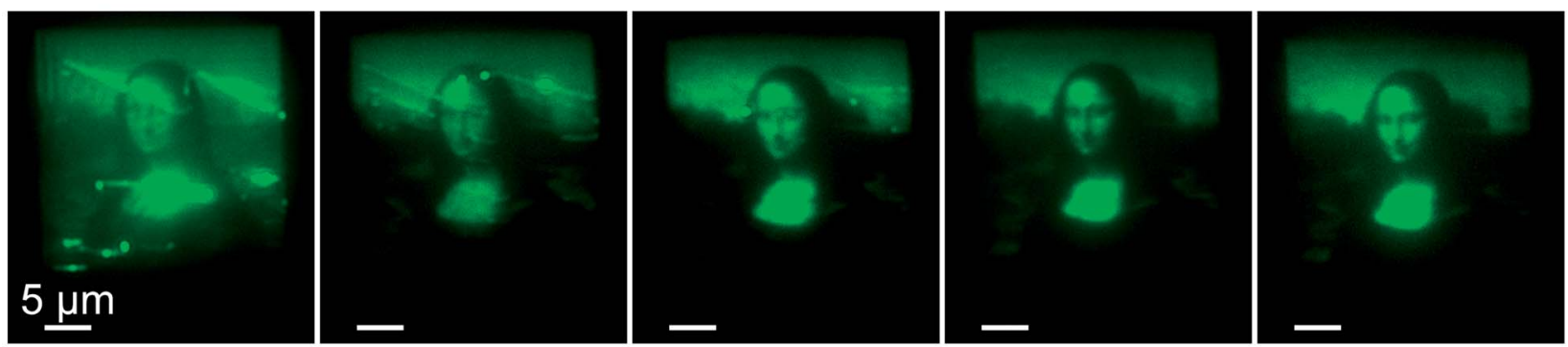

(d)

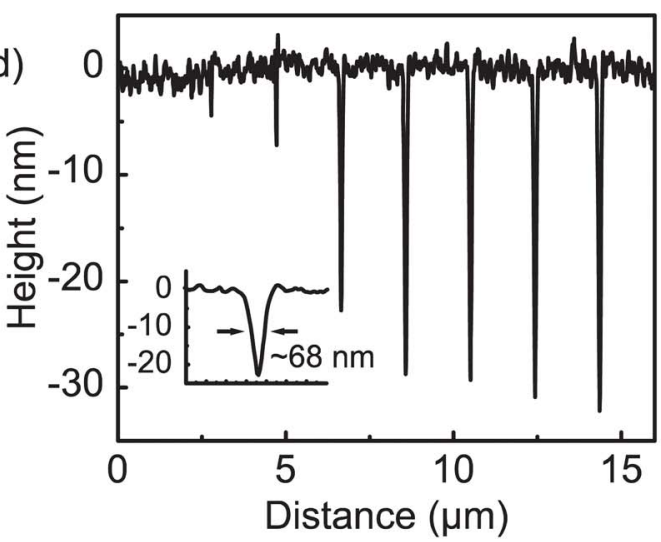

(e)

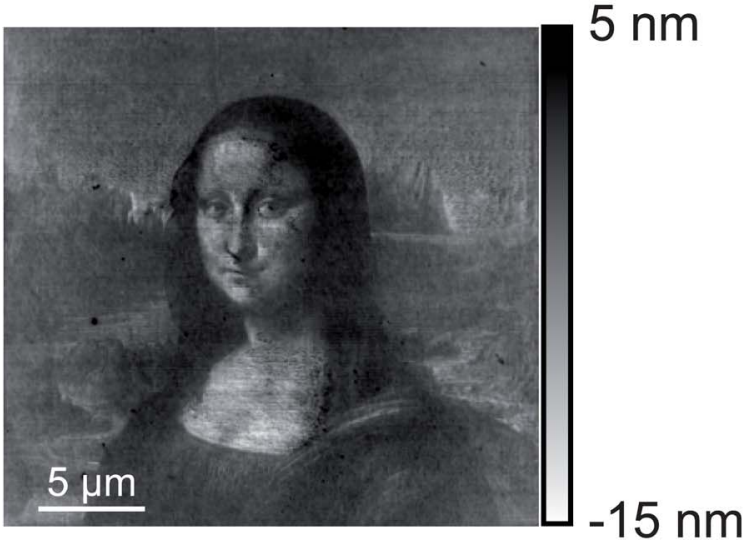

Fig. 2 (a) Five fluorescence images of a series of PPV nanowires written with increasing (left to right) tip temperature, and reproduced five times by using the five tips array. (b) Corresponding AFM topography image of the same PPV wires. (c) Five fluorescence images of a PPV Mona Lisa obtained by using the five tips array. The fluorescence contrast in each Mona Lisa was produced by controlling and varying the temperature of the tips during writing. (d) Topographic cross section of the PPV nanowires produced by tip 1, as shown in (b). (e) AFM topography image of one PPV Mona Lisa image. 
(a)

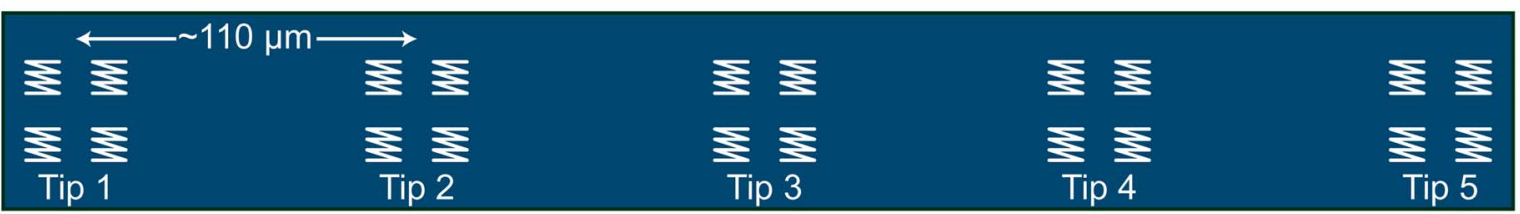

(b)

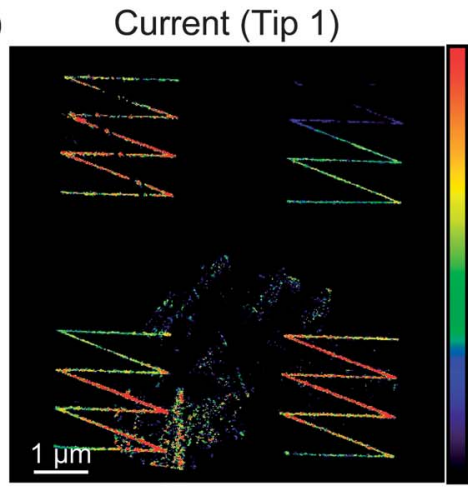

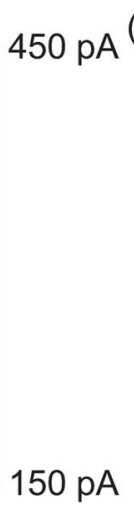

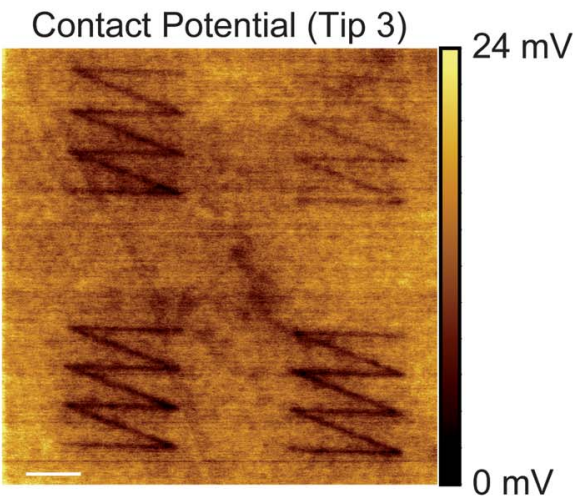

Fig. 3 Parallel TCNL of reduced GO nanostructures. (a) Schematic of parallel TCNL used to produce conductive rGO nanostructures in an insulating GO film. (b) Current and (c) Kelvin probe AFM contact potential difference for rGO nanostructures made by tip 1 and tip 3.

array on a PPV precursor films. The green color contrast has been adjusted individually for each Mona Lisa picture. As before, we remark that the parallelization is not perfect, however it can be substantially improved by programming the temperature (voltage) of each cantilever independently. To demonstrate the level of TCNL control on chemistry and topography, we show in Fig. 2d a cross-section of the topography image of the PPV wires produced by tip 1 (Fig. 2d), clearly confirming the fact that by increasing the tip temperature (left to right) more precursor material is converted in PPV and therefore the resulting indented PPV pattern/wire is deeper. The inset of Fig. $2 \mathrm{~d}$ shows a zoom-in of one of the lines, presenting a full width at half maximum of about $68 \mathrm{~nm}$. This result shows that TCNL arrays can produce sub- $100 \mathrm{~nm}$ lines over distances of $500 \mu \mathrm{m}$. The cross section in Fig. $2 \mathrm{~d}$ shows that by increasing the tip temperature not only more precursor undergoes a chemical change, but also the topography (depth of the indent) can be accurately controlled. This high level of topographical control is demonstrated in Fig. 2e where we report the AFM image of a PPV Mona Lisa pattern.

Finally, the versatility of TCNL arrays is demonstrated by using the very same array used for writing PPV luminescent nanowires and complex topography patterns (Fig. 1 and 2) also to write conductive nanowires of reduced graphene oxide. TCNL can locally reduce highly insulating graphene oxide (GO) to a conductive graphene-like material (here called reduced GO, rGO), as shown in Fig. 3. The TCNL array was therefore used to write graphene-like conductive zig-zag nanostructures in a highly insulating graphene oxide thin film deposited on silicon oxide. Schematic of the experiment is shown in Fig. 3a, where we use each of the five tips to pattern four zig-zag lines at four different temperatures, increasing from top left corner clockwise (see ESI $\dagger$ ). Fig. 3b shows a current sensing-AFM (CSAFM) image of the current flowing between the AFM tip and the GO sample, where the image is focused on the patterns made by tip
1. The reduced GO zig-zag lines conduct more current; this is indicative of the higher conductivity associated with reduced graphene oxide. Fig. $3 \mathrm{c}$ and d show Kelvin Probe Force Microscopy $^{30}$ (KPFM) images of the patterns made by tip 1 and tip 3. These images show a change (between GO and reduced GO lines) in the surface contact potential of $\sim 20 \mathrm{mV}$. The surface contact potential is a measure of the work-function difference between the sample and the tip. The change in conductivity coupled with the change in the surface contact potential is a strong indication that we have successfully reduced graphene oxide with parallel TCNL. In the ESI we report more data regarding the reduction of GO and we also show a high-resolution image of the zig-zag lines indicating a line half maximum width of $50 \mathrm{~nm} . \dagger$

\section{Methods and material section}

We fabricated and used an array of five thermal cantilevers as shown in Fig. 1 and in the ESI. $\dagger$ Each tip is individually addressable and therefore each tip can be heated independently from one another by controlling the electronic current that flows into each cantilever and heats the resistive tip. The design of the array and cantilevers is reported elsewhere ${ }^{27}$ and in the ESI. $\dagger$ This probe array was mounted on a commercial Agilent 5600 LS AFM. One of the key steps for TCNL with an array is to ensure that all the tips are brought into contact with the substrate simultaneously, i.e., all the tips are applying the same load. Under the hypothesis that all the cantilevers have the same spring constant, the load at each tip can be uniformed by aligning the array with the substrate and insuring that all the tips touch the sample simultaneously. This step is achieved (as described in the ESI $\dagger$ ) by measuring each cantilever resistance (which depends on its temperature) as a function of the tipsample distance, because the heat flux (and temperature) in each cantilever dramatically changes as the tip approaches, and 
then touches the sample. ${ }^{31,32}$ Accordingly, an optical leveling support was integrated with the AFM to tilt the substrate in order to level the tips.

The fluorescence images were obtained using a TE2000 Nikon microscope equipped with a 40X, 1.3 NA oil immersed objective and a Roper Scientific CoolSnap CCD camera (Fig. 1).

TCNL on PPV was performed at a linear speed per tip of 10 $\mu \mathrm{m} \mathrm{s}^{-1}$. To produce the 5 Mona Lisa images in Fig. 2 it took 40 minutes in total. TCNL on GO was performed at $0.2 \mu \mathrm{m} \mathrm{s}^{-1}$.

The AFM topography of the PPV nanostructures were obtained with an Agilent 5600 LS AFM (Fig. 2). The scans were performed at scan rates of $16 \mu \mathrm{m} \mathrm{s}^{-1}$. The scans for Fig. $2 \mathrm{~b}$ were made with tip C MikroMasch HQ:NSC35/No Al and 2e was performed with the same thermal tip that made the patterns.

The AFM current and KPFM images of graphene oxide were obtained with a Veeco Nanoscope IV AFM (Fig. 3). The scans were performed at scan rates of $6.4 \mu \mathrm{m} \mathrm{s}{ }^{-1}$ using "NanoAndMore" Pt/Ir coated tips. The current image was obtained by imaging the tip-sample current while a $1.2 \mathrm{~V}$ DC bias was applied between the tip and the sample. The Kelvin probe AFM images ${ }^{30}$ of the contact potential differences were acquired in lift mode with an AC tip-sample bias. The amplitude of the AC bias was $5.5 \mathrm{~V}$, and the frequency was near the mechanical resonance frequency of the cantilever.

PPV precursor films were deposited on silicon substrates. Silicon was cut into $1 \times 1 \mathrm{in}^{2}$ samples; they were scrubbed and rinsed with ethanol. The samples were then Piranha cleaned (3: 1 sulfuric acid to hydrogen peroxide) for about 30 minutes. The samples were placed in a water bath for 30 minutes, and subsequently rinsed with ethanol and nitrogen dried. For most samples, the PPV precursor was spun cast at rates between 300 and $600 \mathrm{rpm}$ and an acceleration of $330(0.5 \mathrm{~mL}$ at a concentration of $0.25 \%$ ). The samples were air dried to remove any remaining precursor solvent. For the Raman measurements, the samples were drop cast instead of spin casting.

The graphene oxide (GO) film (thickness $\sim 50 \mathrm{~nm}$ ) was prepared by drop casting a colloidal GO dispersion on a Si chip and leaving it to dry at $70{ }^{\circ} \mathrm{C}$. Stable colloidal GO dispersions were produced by the modified Hummers method. ${ }^{33}$ More details are reported in the ESI. $\dagger$

\section{Reproducibility and challenges}

The ultimate resolutions obtained by parallel TCNL for the different materials are close to the previously reported resolutions achieved for a single tip. The speed of writing for each individual tip depends on the type of chemical reaction and it does not change when using a single tip or an array.

The major challenge for parallel TCNL with an array of tips is the control of the load across individual cantilevers, because different loads, i.e. contact resistance, correspond to different heat fluxes at the tip-sample contact. ${ }^{34,35}$ Leveling of the array in respect to the substrate improves dramatically the quality of TCNL writing. This step is achieved (as described in the ESI and in the Methods and materials Section $\dagger$ ) by measuring the cantilever resistance while tilting the sample holder. However, the alignment cannot solve completely this issue because the tips can have slightly different height. In the ESI we indeed show that the alignment can bring the tips to touch the sample simultaneously within a range of about $200 \mathrm{~nm}$ (Fig. S2 in the ESI $\dagger$ ). This is particularly evident in Fig. 2, where tip 2 is delivering the least heat because it is applying a lower load than the other tips. To further correct this issue and insure the same heat flux at the tip-sample contact, it will be possible in the future to calibrate the temperature of each tip such that if one tip is applying a lower load than the other tips, then a higher temperature can be delivered to that particular tip. This is possible because each tip can be individually heated.

Another important challenge for parallel TCNL is the alignment of the tips with respect to a desired set of features on the sample, e.g. electrodes. To overcome this issue, it will be possible to couple thermal sensing imaging with the individual addressability of the cantilevers. Thermal sensing imaging can provide a topography map at each tip location, afterwards, by controlling the heat delivered at each tip it will be possible to create TCNL patterns in the desired locations.

\section{Conclusions}

In conclusion we have demonstrated the parallelization of TCNL for nanomanufacturing and imaging arbitrary shaped nano- and micro-structures of semiconducting PPV, and conductive reduced graphene oxide. The same array and set-up can be used interchangeably for the nanofabrication of both materials, requiring only a change in the initial sample, i.e. PPV precursor gel or insulating graphene oxide. Resolution down to sub-50 nm over areas of $500 \mu \mathrm{m}$ and 3D-patterning were achieved. The array can now be extended to other TCNL applications such as biomolecules patterning, chemical nanogradients and growth of complex oxide nanostructures.

\section{Acknowledgements}

This work has been supported by the National Science Foundation CMMI 1100290 (E.R., W.P.K), MRSEC program DMR 0820382 (E.R., J.E.C.), PHYS 0848797 (J.E.C.), the Office of Basic Energy Sciences DOE DE-FG02-06ER46293 (E.R.), the Cariplo Foundation 2011-0373 (L.P., R.S.), and PRIN project GRAF (R.S.).

\section{Notes and references}

1 H. M. Saavedra, T. J. Mullen, P. P. Zhang, D. C. Dewey, S. A. Claridge and P. S. Weiss, Rep. Prog. Phys., 2010, 73, 036501.

2 B. D. Gates, Q. B. Xu, M. Stewart, D. Ryan, C. G. Willson and G. M. Whitesides, Chem. Rev., 2005, 105, 1171-1196.

3 A. A. Tseng, A. Notargiacomo and T. P. Chen, J. Vac. Sci. Technol., B: Microelectron. Nanometer Struct.-Process., Meas., Phenom., 2005, 23, 877-894.

4 R. V. Martinez, N. S. Losilla, J. Martinez, Y. Huttel and R. Garcia, Nano lett., 2007, 7, 1846-1850.

5 R. F. Service, Science, 1996, 274, 1834-1836. 
6 S. A. Maier, M. L. Brongersma, P. G. Kik, S. Meltzer, A. A. G. Requicha and H. A. Atwater, Adv. Mater., 2001, 13, 1501-1505.

7 R. Garcia, R. V. Martinez and J. Martinez, Chem. Soc. Rev., 2006, 35, 29-38.

8 G. M. Whitesides, Nat. Biotechnol., 2003, 21, 1161-1165.

9 P. Vettiger, G. Cross, M. Despont, U. Drechsler, U. Durig,

B. Gotsmann, W. Haberle, M. A. Lantz, H. E. Rothuizen, R. Stutz and G. K. Binnig, EEE Trans. Nanotechnol., 2002, 1, 39-55.

10 K. Salaita, Y. Wang, J. Fragala, R. A. Vega, C. Liu and C. A. Mirkin, Angew. Chem., 2006, 118, 7378-7381.

11 S. C. Minne, J. D. Adams, G. Yaralioglu, S. R. Manalis, A. Atalar and C. F. Quate, Appl. Phys. Lett., 1998, 73, 17421744.

12 R. Szoszkiewicz, T. Okada, S. C. Jones, T. D. Li, W. P. King, S. R. Marder and E. Riedo, Nano Lett., 2007, 7, 1064-1069.

13 D. Wang, R. Szoszkiewicz, M. Lucas, E. Riedo, T. Okada, S. C. Jones, S. R. Marder, J. Lee and W. P. King, Appl. Phys. Lett., 2007, 91, 243104.

14 Z. Wei, D. Wang, S. Kim, S. Y. Kim, Y. Hu, M. K. Yakes, A. R. Laracuente, Z. T. Dai, S. R. Marder, C. Berger, W. P. King, W. A. deHeer, P. E. Sheehan and E. Riedo, Science, 2010, 328, 1373-1376.

15 W. K. Lee, M. Haydell, J. T. Robinson, A. R. Laracuente, E. Cimpoiasu, W. P. King and P. E. Sheehan, ACS Nano, 2013, 7, 6219-6224.

16 S. Kim, Y. Bastani, H. D. Lu, W. P. King, S. Marder, K. H. Sandhage, A. Gruverman, E. Riedo and N. BassiriGharb, Adv. Mater., 2011, 23, 3786-3790.

17 D. Wang, S. Kim, W. D. Underwood, A. J. Giordano, C. L. Henderson, Z. T. Dai, W. P. King, S. R. Marder and E. Riedo, Appl. Phys. Lett., 2009, 95, 233108.

18 O. Fenwick, L. Bozec, D. Credgington, A. Hammiche, G. M. Lazzerini, Y. R. Silberberg and F. Cacialli, Nat. Nanotechnol., 2009, 4, 664-668.

19 A. A. Milner, K. Zhang and Y. Prior, Nano Lett., 2008, 8, 20172022.

20 J. Duvigneau, H. Schonherr and G. J. Vancso, Langmuir, 2008, 24, 10825-10832.
21 D. Pires, J. L. Hedrick, A. De Silva, J. Frommer, B. Gotsmann, H. Wolf, M. Despont, U. Duerig and A. W. Knoll, Science, 2010, 328, 732-735.

22 D. Wang, V. K. Kodali, W. D. Underwood, J. E. Jarvholm, T. Okada, S. C. Jones, M. Rumi, Z. Dai, W. P. King, S. R. Marder, J. E. Curtis and E. Riedo, Adv. Funct. Mater., 2009, 19, 1-7.

23 K. M. Carroll, A. J. Giordano, D. B. Wang, V. K. Kodali, J. Scrimgeour, W. P. King, S. R. Marder, E. Riedo and J. E. Curtis, Langmuir, 2013, 29, 8675-8682.

24 A. W. Knoll, D. Pires, O. Coulembier, P. Dubois, J. L. Hedrick, J. Frommer and U. Duerig, Adv. Mat., 2010, 22, 33613365.

25 D. Pires, J. L. Hedrick, A. De Silva, J. Frommer, B. Gotsmann, H. Wolf, M. Despont, U. Duerig and A. W. Knoll, Science, 2010, 328, 732-735.

26 R. H. Rice, P. Mokarian-Tabari, W. P. King and R. Szoszkiewicz, Langmuir, 2012, 28, 13503-13511.

27 H. J. Kim, Z. Dai and W. P. King, J. Micromech. Microeng., 2013, 23, 025001.

28 B. A. Nelson and W. P. King, Nanosc. Microsc. Therm., 2008, 12, 98-115.

29 D. Wang, S. Kim, W. D. Underwood II, A. J. Giordano, C. L. Henderson, Z. Dai, W. P. King, S. R. Marder and E. Riedo, Appl. Phys. Lett., 2009, 95, 233103-233108.

30 M. Nonnenmacher, M. P. Oboyle and H. K. Wickramasinghe, Appl. Phys. Lett., 1991, 58, 2921-2923.

31 W. P. King and K. E. Goodson, J. Heat Transfer, 2007, 129, 1600-1604.

32 W. P. King, T. W. Kenny, K. E. Goodson, G. L. W. Cross, M. Despont, U. T. Durig, H. Rothuizen, G. Binnig and P. Vettiger, J. Microelectromech. Syst., 2002, 11, 765-774.

33 W. S. Hummers and R. E. Offeman, J. Am. Chem. Soc., 1958, 80, 1339.

34 K. M. Carroll, A. J. Giordano, D. Wang, V. K. Kodali, J. Scrimgeour, W. P. King, S. R. Marder, E. Riedo and J. E. Curtis, Langmuir, 2013, 29, 8675-8682.

35 M. A. Lantz, B. Gotsmann, U. T. Durig, P. Vettiger, Y. Nakayama, T. Shimizu and H. Tokumoto, Appl. Phys. Lett., 2003, 83, 1266-1268. 